\title{
Controle de dopagem no esporte: aspectos químicos e farmacológicos que afetam a detecção de drogas no cabelo
}

\author{
Francisco Radler de Aquino Neto ${ }^{1 *}$, Marlice Aparecida Sipoli Marques ${ }^{1,2}$, Henrique Marcelo \\ Gualberto Pereira ${ }^{1}$ \\ ${ }^{1}$ LABDOP, Instituto de Química, Departamento de Química Orgânica, Universidade Federal do Rio de Janeiro, \\ ${ }^{2}$ LABDOP, Instituto de Química, Departamento de Química Analítica, Universidade Federal do Rio de Janeiro
}

*Correspondência:

F. R. Aquino Neto

Instituto de Química/UFRJ

Centro de Tecnologia - BI. A - Sala 607

Ilha do Fundão - Cidade Universitária

21949-9000 - Rio de Janeiro - RJ

E-mail: radler@iq.ufrj.br
Análise em cabelo (AC) é bem documentada na área de toxicologia forense. AC tem sido empregada para inferir se o consumo de determinado fármaco é crônico ou esporádico. A Sociedade de Teste Capilar (STC) publicou normas sobre AC em dopagem no esporte, às quais são aceitas em cortes judiciais, apesar de não terem sido incorporadas pelo Comitê Olímpico Internacional (COI). Dentre as substâncias proibidas pelo COI o grande desafio da AC na área de controle de dopagem (CD) é a comprovação da sua viabilidade na detecção de esteróides anabolizantes (EA). Antes de validar a AC para o CD, a comunidade científica tem de responder pelo menos a cinco questões críticas: Qual a quantidade mínima detectável no cabelo após a administração? Qual a relação entre a quantidade detectada e a encontrada no cabelo? Qual a influência da cor do cabelo? Existe algum viés no teste em cabelo? Qual a influência da contaminação exógena e do tratamento capilar e com cosmético? O fator limitante da AC em dopagem de atletas é a carência de dados científicos. O presente artigo faz uma revisão dos trabalhos publicados com um enfoque aos parâmetros analíticos e farmacológicos que limitam o emprego da AC no CD.

\section{INTRODUÇÃO}

\section{Controle de dopagem e sua relação com análises forenses}

A análise de cabelo (AC) é de grande aceitação em toxicologia forense (Grahan et al., 1989; Kintz, Mangin, 1993; Cone et al., 1994; Kintz, 1996a; Chiarrotti et al., 1996; Baungartner, Hill, 1993; Kintz, Samyn, 1999; Mieczkowski, 1999; Cone, 2001; Verstraet, 2001). Embora a AC não seja aprovada pelo Comitê Olímpico Internacional (COI), nos últimos anos ela vem sendo aceita em inúmeras cortes de justiça. Tal fato deve-se à grande expe- riência adquirida pelos laboratórios forenses, onde, por mais de 30 anos, na Europa, os toxicologistas foram chamados a analisar amostras de produtos encontrados no mercado, de sangue, vísceras e urina ligadas às áreas esportiva e policial (Uematsu, Sato, 1990; Offidani, 1993; Tracqui, 1995; Pepin, Gaillard, 1997; Tracqui et al., 1997; Perkoning et al., 1998; Pragst et al., 1998; Vicent et al., 1999; Kintz, Samyn, 1999; Quintella, 2000). Um dos casos mais famosos em que a toxicologia forense prestou auxilio ao CD foi na elucidação da causa "mortis" de Tom Simpson no Tour da France, em 1967 (Laure, 1997). Após uma autópsia (suco gástrico, urina e sangue) comprovouse a morte por abuso de anfetamina. Em 1998, a mesma 
substância foi encontrada nas vísceras de um jogador de futebol (Kintz et al., 1999b). Nos últimos dois anos pesquisadores dessa área vêm analisando os cabelos da cabeça e pubianos para elucidar a causa de morte ou indicar se uma pessoa é traficante e/ou usuário de drogas (Henderson et al., 1993; Cone et al., 1993; Kintz, Mangin, 1995; Cirimele et al., 1996; Henderson et al., 1998; Deveaux et al., 2000).

$\mathrm{Na}$ área de alimentos (controle de anabolizantes em animais de abate) a análise do pêlo vem sendo utilizada na monitoração de mais de 24 resíduos de anabolizantes (Sphon, 1961; Stephany, Van Ginkel, 1990; Moller, Fey, 1992; Gaillard et al., 1997; Gaillard et al., 1999a; Sawaya et al., 2000; Carrasquilla, 2001).

\section{Controle de dopagem em diferentes matrizes}

O COI defende, a longo tempo, o controle de dopagem com base em três princípios: (1) proteção da saúde do atleta, (2) defesa da ética médica e esportiva e (3) igualdade de chances para todos os esportistas (De Merode, 1999; The OMAC, 2001). Para um melhor controle, o COI divulgou uma listagem com 128 substâncias de uso proibido, enfatizando a proibição de substâncias similares (em relação tanto à estrutura química quanto aos efeitos farmacológicos) (The OMAC, 2001; Schänzer, 2001).

A concentração urinária da maioria dessas substâncias (denominadas clássicas) excede os valores encontrados em outras matrizes biológicas, tornando essa matriz de fácil coleta a eleita para controle de dopagem (Delbeke, 1991; Donike et al., 1992; Shanzer, Donike, 1993; Ayotte et al., 1996; Hemmersbach, De La Torre, 1996; Geyer et al., 1998; Horning et al., 1998a-b; Ramos et al., 1998; Marques et al., 1999; Ramos, 2000; Henze et al., 2001; Aquino Neto, 2001; Pereira et al., 2001). Nos últimos anos, o advento de novas substâncias, como hCG, IGF-1, EPO (eritropoietina), interleucina-3, PFCs, expansor do volume plasmático e pró-hormônios, impulsionou não apenas a busca de novas metodologias analíticas, mas também o estudo de matrizes alternativas para o controle das mesmas (Breo, 1990; Rickert et al., 1992; Saugy et al., 1996 ; Schänzer et al., 1996; Healy et al., 1997; Abranson et al., 1996; Audran et al., 1999; Augenstein, 1999; Birkland, Hemmersbach, 1999; Bôer et al., 1999; Browne et al., 1999; Riess, et al., 1999; Callies et al., 2000; Carrasquilla, 2000; Geyer et al., 2000; Thevis et al., 2000; De Cock et al., 2001; Draisci et al., 2001; Saugy, 2001).

O uso de pró-hormônios pode ser caracterizado através da detecção dos seus metabólitos na urina (Geyer et al., 2000; Schänzer et al., 2000). Entretanto, tal fato não permite a elucidação inequívoca da origem dos mesmos. Desta forma, não se distingue, por exemplo, se houve a ingestão dos pró-hormônios, ou se o consumo foi do fármaco (Shackelton et al., 1997). Tal distinção pode ser alcançada através da AC, uma vez que o alvo analítico é o fármaco não-biotransformado (Kintz, 1998) (Tabelas I e II).

Para as substâncias, denominadas não-clássicas (ver texto anterior), matrizes alternativas como cabelo, sangue, plasma e ar expirado devem ser investigados (Horning et al., 1977; Swinkels et al., 1992; Lac et al., 1993; Dongmi et al., 1996; Ciremille et al., 1999; Gaillard et al., 1999; Gaillard et al., 1999; Ciremelle et al., 2000;) à semelhança do que acontece em análise de drogas de abuso (Cone, 2001; Fucci, 2001). O sangue devido a questões de ordem religiosa e por necessitar uma coleta invasiva, não vem sendo muito utilizado, com exceção do teste para a epoetina (Browne et al., 1999, Tabelas I e II). O ar expirado é a matriz de escolha no controle de PFCs, os quais também podem ser detectados no sangue por extração lí-

TABELA I - Comparação entre as análises de anfetaminas em urina, suor, saliva e cabelo

\begin{tabular}{lllll}
\hline Parâmetro & Urina & Suor & Saliva & Cabelo \\
\hline coleta da amostra & privacidade & não-invasiva & não-invasiva & não-invasiva \\
& na coleta & & & \\
fármaco-mãe e & fármaco-mãe & fármaco-mãe & fármaco-mãe \\
metabólitos & $>>$ metabólitos & $>$ metabólitos & > metabólitos \\
janela de detecção & $2-3$ dias & 1 semana & algumas horas & alguns meses \\
concentração do fármaco & alta & baixa & baixa & baixa \\
problemas associados & adulteração & amostra limitada & amostra limitada & contaminação \\
& & & & ambiental \\
unidade & ng/mL & ng/pach & ng/mL & ng/mg \\
\hline
\end{tabular}

Modificado de Kintz, Samyn, 1999. 
TABELA II - Comparação entre as matrizes urina e cabelo no controle de dopagem

\begin{tabular}{lll}
\hline Parâmetros & Urina & Cabelo \\
\hline fármaco & todos, exceto alguns peptídeos & todos exceto hormônios \\
analito & metabólitos & fármaco-mãe \\
período de detecção & 2 dias, exceto anabolizantes & semanas - meses \\
tipo de medida & aumentada & acumulativa \\
triagem & sim & não \\
invasividade & alta & baixa \\
estocagem & $-20{ }^{\circ} \mathrm{C}$ & temperatura ambiente \\
risco de falso-negativo & alta & baixa \\
risco de falso-positivo & baixa & não foi determinado \\
risco de adulteração & sim & baixo \\
controle do material & necessário & necessário \\
\hline
\end{tabular}

Modificado de Kintz, 1998.

quido-líquido (Breo, 1990; Audran et al., 1999; Birkland, Hemmersbach, 1999; Bôer et al., 1999; Krafft, 2001). Saliva ou suor não são matrizes de escolha para as novas substâncias (Schraman et al.; 1992, Cone, 1993) (Tabelas I e II). Dentre essas matrizes, o cabelo, devido ao seu uso histórico em análise forense, poderá vir a ser empregado na análise de dopagem, desde que algumas questões de ordem farmacológica e analítica sejam esclarecidas com base em dados científicos (Gleixner et al., 1996; Hold et al., 1996; Kintz, 1998; Wheeler et al., 1998; Deng et al., 1999; Thieme et al., 1999).

\section{Qual a quantidade mínima detectável de fármacos no cabelo?}

Nos últimos dois anos, uma das grandes controvérsias acerca da utilização do cabelo como matriz no controle de dopagem (particularmente no caso da nandrolona, estanozolol, corticosteróides e alguns $\beta_{2}$-agonistas) (Hold et al., 1996; Madea et al., 1998; Sherer et al., 1998; Wheeleer et al., 1998; Kintz et al., 1999b,c; Machnick et al., 1999; Thieme et al., 1999; Draisci et al., 2001; Kintz et al., 2001) é a possibilidade das análises em urina configurarem um resultado positivo, enquanto que, simultaneamente, as análises em cabelo resultarem em laudo negativo (Rivier, 2000). A questão relevante é se o procedimento analítico adotado na análise do cabelo tinha sensibilidade suficiente para detectar baixas concentrações. Segundo Gaillard (1999a, b), tal problema foi devido à falta de sensibilidade das técnicas empregadas (Gaillard et al., 1997, 1999a, b; Kintz et al., 2001; Wu, 1999) e não à matriz (cabelo). Wu (1999), em um estudo empregando diferentes procedimentos analíticos, comprovou que é possível detectar no cabelo, nandrolona de dois a seis meses após a administração de $50 \mathrm{mg}$ de decanoato de nandrolona. Tais resultados foram compatíveis com o encontrado na urina, onde foram observados os metabólitos norandrosterona e noretiocolanolona até oito meses após a administração. Esses resultados são conflitantes com os de Segura et al. (1999), que não detectaram a presença de nandrolona após a administração de uma única dose, do decanoato das referidas substâncias, embora os autores tenham reportado aumento na concentração de testosterona. Cirimele et al. (1999a, 2000), em um estudo com estanozolol, identificaram apenas o fármaco-mãe no cabelo de fisiculturistas, ao contrário do obtido por Hold et al. (1996) e Thieme et al. (1999), que também detectaram o 3 'OH-estanozolol. Os autores concluíram que tais diferenças eram devido à dose administrada, já que a razão entre os dois metabólitos era de 30:1.

Por outro lado, Kintz (1998), empregando técnica de detecção mais sensível, conseguiu comprovar, através de um estudo comparando AC e urina em controle de dopagem, que o número de falso-negativos era menor que com as técnicas convencionais. $\mathrm{O}$ autor relatou que uma das grandes vantagens da AC era a identificação dos falso-negativos em relação à análise de urina (Tabela II). Ressalta-se que o período de detecção de dois dias (exceto para anabolizantes) descrito pelo autor diz respeito não à incapacidade de detecção em períodos mais longos e sim à falta de relação do consumo de certos fármacos (como por exemplo estimulantes) até 48 horas antes da competição e a intenção de dopagem.

As propriedades físico-químicas dos fármacos são de fundamental importância na elucidação da sua taxa de incorporação no cabelo. Nakahara (1995) e Nakahara et 
al., (1995) investigaram fatores como a afinidade dos fármacos pela melanina, assim como a lipofilicidade e permeabilidade dos mesmos na membrana capilar. Fármacos neutros (esteróides, benzodiazepínicos, canabinóides, entre outros) ou ácidos têm uma baixa incorporação capilar, ao contrário de fármacos básicos (cocaína, anfetamina, etc.). $\mathrm{O}$ autor concluiu que como os esteróides são altamente lipofílicos e neutros, a permeabilidade na membrana depende do gradiente de $\mathrm{pH}$ entre o sangue ( $\mathrm{pH} 7,4)$ e a matriz ácida (cabelo, $\mathrm{pH} \sim 5,0$ ). Isto explicaria o porquê da baixa concentração dos esteróides no cabelo, normalmente relatada na literatura, e, conseqüentemente, os resultados negativos quando o atleta faz uso de uma única dose. Um fato interessante é que segundo essa teoria o fármaco-mãe e/ou seus metabólitos após a administração, biotransformação e distribuição estariam presentes no sangue, portanto, teriam a possibilidade de se difundirem (difusão passiva) para o cabelo (Moller, 1992; Springfield, 1993; Staub, 1993; March, Evans, 1994; Cone, 1996; Kintz et al., 1996a-b; Kircher, Parlar, 1996; Rollins et al., 1996; Weeller et al., 1998; Kintz, Samyn, 1999). Portanto, é de se esperar que tanto a fármaco inalterado quanto o(s) seu(s) metabólito(s) seja $(\mathrm{m})$ detectados na análise capilar. Entretanto, no caso dos anabolizantes, observa-se, preferencialmente o fármaco inalterado, sendo que o(s) seu(s) metabólito(s) em muitos estudos nem foram detectados. Essa observação mais uma vez aponta a importância da lipofilicidade no acúmulo dos anabolizantes no cabelo, já que a biotransformação confere aos metabólitos polaridade maior que o do fármaco inalterado.

No caso dos $\beta_{2}$-agonistas, o mecanismo de interação com a melanina do cabelo envolveria interações eletrostáticas, uma vez que a comparação entre o clembuterol (amina primária) e o salbutamol mostrou parâmetros de distribuição diferenciados, sendo que o acúmulo do primeiro no cabelo é dez vezes maior. Schänzer et al. (1996), após a administração de uma única dose de clembuterol, também detectaram a presença do mesmo em cabelo e urina por CGEMAR.

Howells et al. (1994) determinaram a capacidade da ligação do clembuterol e da nandrolona à melanina. Os autores encontraram um valor de 5,9 nmol. $\mathrm{mg}^{-1} \mathrm{e}$ $0,59 \mathrm{nmol} \cdot \mathrm{mg}^{-1}$, para o clembuterol e a nandrolona, respectivamente, comprovando que no caso de fármacos básicos, como o clembuterol, a afinidade das mesmas pela melanina seria superior à observada com o fármaco neutro (nandrolona). Conseqüentemente, até que uma intercomparação valide as duas matrizes, muito cuidado deve ser tomado na comparação dos dados obtidos em urina e na $\mathrm{AC}$.

Gallaird et al. (1997, 1999a, b) determinaram que o baixo número de resultados positivos para corticosteróides se devia à não implementação de triagem dessas substâncias em análise de rotina nos laboratórios do $\mathrm{COI}$ e, no caso dos esteróides anabolizantes, se devia não só à falta de utilização de técnicas sensíveis como reportado por Schänzer et al., (1996), mas também à impossibilidade de empregar a matriz (urina) após ter decorrido longo período da administração (Tabelas III-V). Os autores comprovaram, através da $\mathrm{AC}$, que ciclistas profissionais faziam uso dos glicocorticosteróides (CT) triancinolona, betametasona, prednisolona e hidrocortisona. No caso da triagem de anabolizantes em cabelo, os autores observaram que apesar desses apresentarem baixa afinidade por essa matriz, o número de resultados positivos aumentava de $8 \%$, se comparado ao observado na triagem em urina desde que uma técnica como a CGEMAR fosse empregada.

TABELA III - Dados estatísticos dos resultados positivos relatados pelos laboratórios olímpicos nos anos de 1991 - 1996

\begin{tabular}{lrrrrrr}
\hline Classe de substâncias e número & \multicolumn{7}{c}{ Ano } \\
\cline { 2 - 7 } de amostras analisadas & $\mathbf{1 9 9 1}$ & $\mathbf{1 9 9 2}$ & $\mathbf{1 9 9 3}$ & $\mathbf{1 9 9 4}$ & $\mathbf{1 9 9 5}$ & $\mathbf{1 9 9 6}$ \\
\hline Número de amostras analisadas & 84088 & 87808 & 89166 & 93680 & 93938 & 96454 \\
Número de positivos & 904 & 1204 & 1488 & 1301 & 1283 & 1263 \\
Estimulantes & 221 & 277 & 339 & 347 & 310 & 281 \\
Narcóticos & 72 & 102 & 48 & 42 & 34 & 37 \\
Anabolizantes & 552 & 717 & 995 & 823 & 854 & 881 \\
Diuréticos & 47 & 70 & 66 & 63 & 59 & 54 \\
Hormônios peptídicos & 1 & 4 & 4 & 3 & 9 & 4 \\
Agentes mascaradores & 1 & 22 & 23 & 8 & 3 & 0 \\
$\beta$-bloqueadores & 10 & 2 & 13 & 15 & 14 & 6 \\
\hline
\end{tabular}

Modificado de Schänzer, 2001. 
TABELA IV - Resultados relatados por Gaillard et al. (1999a, 2000) em urina e cabelo para esteróides anabolizantes

\begin{tabular}{lccc}
\hline Resíduos identificados & N & $\begin{array}{c}\text { Urina } \\
(\mathbf{n g} / \mathbf{m L})\end{array}$ & $\begin{array}{c}\text { Cabelo } \\
(\mathbf{n g} / \mathbf{m g})\end{array}$ \\
\hline Nandrolona & 1 & 0,0 & 5,1 \\
Undecanoato de testosterona & 2 & 0,0 & 15,2 \\
& $3-25$ & 0,0 & 0,0 \\
& $26-30$ & 0,0 & $\mathrm{QI}$ \\
\hline Número total de positivos & 0 & 2 \\
Número de análise & 30 & 25 \\
Percentagem total de positivos & 0 & 8 \\
\hline
\end{tabular}

QI: Quantidade insuficiente.

TABELA V - Resultados relatados por Gaillard et al. (1999b, 2000) em urina e cabelo para corticosteróides

\begin{tabular}{|c|c|c|c|}
\hline Resíduos identificados & $\mathbf{N}$ & $\begin{array}{c}\text { Urina } \\
(\mathrm{ng} / \mathrm{mL})\end{array}$ & $\begin{array}{c}\text { Cabelo } \\
\text { (ng/mg) }\end{array}$ \\
\hline Triancinolona acetonida & $\begin{array}{c}1 \\
2 \\
3 \\
4 \\
5 \\
6 \\
7 \\
8 \\
9 \\
10\end{array}$ & $\begin{array}{l}4,5 \\
3,2 \\
2,4 \\
2,3 \\
2,2 \\
1,8 \\
1,8 \\
1,7 \\
1,5 \\
1,1\end{array}$ & $\begin{array}{c}\text { QI } \\
0,0 \\
0,14 \\
\text { QI } \\
\text { QI } \\
\text { QI } \\
\text { QI } \\
0,22 \\
0,33 \\
0,0\end{array}$ \\
\hline Betametasona & $\begin{array}{l}11 \\
12\end{array}$ & $\begin{array}{c}18,2 \\
0,9\end{array}$ & $\begin{array}{l}\text { QI } \\
\text { QI }\end{array}$ \\
\hline Acetato de hidrocortisona & 13 & 0,0 & 1,31 \\
\hline Metilprednisolona & $\begin{array}{c}14 \\
15-19 \\
20-30\end{array}$ & $\begin{array}{l}0,0 \\
0,0 \\
0,0\end{array}$ & $\begin{array}{c}1,21 \\
0,0 \\
\text { QI }\end{array}$ \\
\hline Número total de positivos & & 12 & 5 \\
\hline Número de análise & & 30 & 12 \\
\hline Percentagem total de positivos & & 40 & 42 \\
\hline
\end{tabular}

QI: Quantidade insuficiente.

Como, ao contrário dos estimulantes, os anabolizantes são cronicamente administrados antes da competição, um estudo longitudinal de acordo com o comprimento do cabelo auxiliaria muito na interpretação dos resulta- dos. À semelhança do que ocorre no caso do abuso de cocaína, a comparação dos resultados da análise entre urina e AC permitiram confirmar se um atleta havia realmente consumido um copo de chá de coca (contendo 3,9 $\mathrm{mg}$, tão comum em certas culturas) como foi constatado por Preston (1997).

\section{Qual a relação entre a quantidade detectada do fármaco usado e a concentração da mesma e/ou seus metabólito(s) no cabelo?}

Quando um resultado positivo é relatado, mesmo que o COI não exija uma análise quantitativa, inúmeras perguntas concernentes são colocadas, tais como: (1) A concentração encontrada é alta? O atleta fez uso de uma grande quantidade do fármaco? A quantidade encontrada pode ser relacionada com o tempo decorrido após o uso?

Em relação a $\mathrm{AC}$, duas abordagens podem ser adotadas para responder a essas indagações. A primeira envolve a comparação entre a concentração obtida e os valores (alto, médio ou baixo) observados por usuários declarados. Por exemplo, de acordo com Pepim e Gaillard (1997), a concentração de cocaína no cabelo em valores menores que $4 \mathrm{ng} / \mathrm{mg}$ indica baixo consumo. A segunda compara os resultados com dados obtidos nos estudos de excreção (Quintella et al., 2000).

No caso dos anabolizantes, com exceção da testosterona e DHEA, cujas concentrações são da ordem de 110 pg (Hold et al., 1996; Gaillard et al., 1999a, b; Cirimele et al., 2000; Carrasquilla et al., 2001), não existem dados disponíveis na literatura que permitam estabelecer o padrão de uso.

A concentração normalmente encontrada de drogas de abuso no cabelo é da ordem de ng/mg (Quintella et al., 2000), enquanto que a concentração dos anabolizantes e CT é de pg/mg. No caso da análise do cabelo de fármacos como a cocaína e opiáceos, para que a $\mathrm{AC}$ venha a ser usada no controle de dopagem falta somente estabelecer a concentração de corte (Kintz, 1998). Uma vez que as metodologias em uso são capazes de detectar ng/mg dessas substâncias sem incorrer em resultados inequívocos.

$\mathrm{O}$ grande interesse na $\mathrm{AC}$ vem do fato da possibilidade de estabelecer a história dos últimos meses de exposição a um ou outro xenobiótico e da possível correlação dose/concentração no cabelo, o que não é possível com a utilização de amostras biológicas como o sangue, saliva e urina. Para a cocaína, uma única dose de 25-35 mg permite a sua detecção $2-6$ meses após o consumo. A codeína pode ser detectada 8 semanas após uma única dose de $60 \mathrm{mg}$, enquanto que para os EA e CT, ainda falta estabelecer metodologias de referência. 
Até o presente momento, os dados disponíveis na literatura sobre a concentração desses fármacos (EA, CT) no cabelo são conflitantes. Alguns relatam que há uma correlação entre a quantidade administrada (dosagem) e a concentração encontrada no cabelo. Outros concluem o inverso. A falta de correlação entre os dois parâmetros, dose administrada e concentração no cabelo, poderia ser explicada por vários fatores: variabilidade no ciclo de crescimento capilar, (2) influência no tratamento capilar com cosméticos, (3) práticas de higiene, (3) incerteza na dose ingerida, (4) grau de pureza da substância administrada e (5) taxa de sudorese e variação na secreção sebácea e apócrina entre os indivíduos. Até que estudos controlados sejam realizados (se isto for possível) será difícil empregar a AC para calcular a dose administrada ou estabelecer o tempo transcorrido após a administração.

\section{Qual a influência da cor do cabelo?}

A melanina é a responsável pela cor do cabelo, como determinado pelo teor de fiomelanina e eumelanina presentes na matriz. Cabelos castanhos e pretos têm maior quantidade de eumelanina do que cabelos vermelhos e louros, nos quais predomina a fiomelanina. Estudos comprovam que a ligação dos fármacos ao cabelo se dá através da melanina. Tal fato explica porque o teor é maior em cabelo escuro do que nos demais (Kintz, Samyn, 1999).

Em 1992, Hold et al. (1996) demonstraram que o estanozol se incorpora melhor em cabelos escuros, sendo o mesmo observado para a nandrolona (Hold et al., 1996; Ciremelle et al., 1999b; Gaillard et al., 1999a; Kintz et al., 1999a-c; Ciremelle et al., 2000; Kintz et al., 2001) e clembuterol (Gleixner et al., 1996; Gaillard, 1997; Machnnick et al., 1999). Kronstrand et al. (1999) observaram que a relação entre a concentração de codeína e melanina no cabelo era exponencial. Todos esses resultados apontam que a concentração de melanina é determinante no acúmulo de fármacos no cabelo. Portanto, a cor do cabelo pode ser um viés em teste capilar.

\section{Existe viés racial em análise de cabelo?}

Vários pesquisadores têm demonstrado que diferentes tipos de cabelo incorporam variadas quantidades de fármacos quando expostos às mesmas condições. Esses estudos sugerem que cabelos grossos e negros acumulam mais que os finos e loiros. Henderson et al. (1996) demonstraram que, em condições experimentais idênticas, indivíduos não-caucasianos (n-9) incorporam 3 vezes mais cocaína deuterada no cabelo do que os caucasianos. Cone et al. (1993 e 1994) também obtiveram os mesmos resultados com a população de sul-africanos. Tal fato pode levar a maior número de positivos em uma população do que em outra. $\mathrm{O}$ estabelecimento de uma concentração de corte não resolveria tal problema. Kidwell (1999) propôs que tais diferenças não são devidas à raça, mas aos diferentes cuidados adotados com os cabelos (tratamentos, higiene, hábitos).

\section{Qual a influência de contaminação exógena e do tratamento capilar?}

A estabilidade do fármaco no cabelo pode ser influenciada por procedimentos como permanentes, tinturas, branqueadores e exposição a ultravioleta. De um modo geral, a concentração de um fármaco no cabelo declina 30 a 80\% quando se compara o valor observado antes com aqueles obtidos após esses tratamentos (Henderson, 1993; Cirimele, 1995; Potsch, Skopp, 1996; Jurado et al., 1997; Kidwell, 1999b), podendo levar a falso-negativo. Cirimile et al. (1999) relataram que o uso de xampu contendo canabinóides não leva a resultado positivo. Em caso da não disponibilidade do cabelo da cabeça, recomenda-se a coleta de pelos pubianos ou de outra região, já que com exceção da testosterona e da DHEA a concentração das demais substâncias não difere nos cabelos da região genital, axila, braço ou da cabeça.

Já a contaminação exógena vem sendo reportada na literatura como a principal causa de resultados falso-positivos originados a partir da exposição passiva das substâncias provenientes do meio ambiente e/ou suor (Wenning, 2000). A STC adotou critérios visando auxiliar a redução da incidência de falso-positivos provenientes de contaminação passiva. Critérios como: adoção de procedimentos de descontaminação, emprego de concentrações de corte, avaliação da relação fármaco/metabólitos presentes e identificação dos metabólitos. No caso especifico de alguns fármacos como a cocaína e a heroína, a relação entre as mesmas e seus principais metabólitos já vem sendo usada (benzoilecgonina/cocaína $>0,05$ e 6-monoacetilmorfina/morfina $>1,3$, respectivamente). Carrasquilla (2001) demonstrou que em pêlo animal exposto ao clembuterol foi possível realizar descontaminação prévia com o auxílio de uma solução aquosa ácida, diminuindo o número de falso-positivos.

\section{PERSPECTIVAS E CONCLUSÕES}

No caso de controle de dopagem, a triagem das substâncias proibidas é realizada empregando métodos padrões validados em laboratórios do COI. A corte judicial pode requerer informações adicionais sobre o padrão de uso dessas substâncias, como aconteceu durante o 
"tour" da França, onde o cabelo foi coletado, juntamente com outras matrizes alternativas. O cabelo pode ser usado para confirmar abuso e identificar a natureza exata do fármaco-mãe (por exemplo, nandrolona, norandrostenodiol, norandrostenodiona, em caso positivo de nandrolona). $\mathrm{O}$ uso contínuo de fármacos (vários meses) restritos, como por exemplo o salbutamol, fenoterol, terbutalina e glicocorticosteróides, pode ser documentado por análise em cabelo. A análise de ésteres de testosterona nessa matriz pode auxiliar na discriminação de testosterona exógena.

Entretanto, antes de considerar a AC válida pelo $\mathrm{COI}$ algumas questões precisam ser discutidas (como por exemplo, o tratamento capilar). A relação entre os resultados provenientes da análise na urina e no cabelo, ainda não está bem estabelecida. Deste modo, um resultado negativo de EA no cabelo, não significa resultado negativo de dopagem em atletas. A utilização de métodos padrão empregando técnicas de detecção mais sensíveis para análise de EA em cabelo possibilitaria diminuir o limite de detecção, permitindo a identificação de metabólitos de longa duração, aumentando em muito a janela de detecção nessa matriz à semelhança do que ocorre na análise de cocaína. A discriminação étnica nos resultados de AC precisa ser revista para evitar arbitrariedades no controle de dopagem. Por outro lado, a comprovação em alguns casos de que a contaminação capilar não influencia o resultado de dopagem, permitiria estabelecer se um positivo de THC é ou não proveniente de contaminação passiva, o que não ocorre na urina, onde foi necessário estabelecer uma concentração de corte de $15 \mathrm{ng} / \mathrm{mL}$.

A proibição do uso de diuréticos parece ser cabível apenas em conexão a análises em urina. A intenção de mascarar metabólitos urinários ou suprimir a excreção de algum fármaco não é um critério aplicável a matriz cabelo.

Para efeito de dopagem, as possíveis vantagens decorrentes do abuso de estimulantes, narcóticos e bbloqueadores são limitadas ao intervalo de tempo consistente com as respectivas ações farmacológicas. Deste modo, tais fármacos são monitorados exclusivamente dentro de competições (in competition). Esta estratégia exclui a adoção de uso AC. Uma limitação adicional do uso da $\mathrm{AC}$ envolve a prática de raspagem dos pêlos comuns em esportes aquáticos e já observados também em outras modalidades esportivas.

No caso de AC, o estabelecimento da faixa normal do perfil esteróide endógeno sofre a influência de diversos fatores técnicos e biológicos torna a avaliação consideravelmente mais complexa.

\section{ABSTRACT \\ Doping control in sports: Chemical and pharmacological aspects that effect the detection of drugs in hair}

Hair analysis is very well documented in forensic toxicology. It has been employed for differentiation between chronic or occasional consumption of certain drugs. The Society of Hair Testing published rules about hair analysis in sportive doping, which are accepted in most courts of justice, in spite that they had not been incorporated by the International Olympic Committee. Among the substances forbidden by the IOC, the great challenge of hair analysis in doping control is to confirm his validity in the detection of anabolic steroids. Before validation of hair analysis in doping control, the scientific community has to answer at least five critical questions: (1) What is the minimal amount detectable in hair after administration? (2) What is the relationship between the amount of the drug used and the concentration of the drug or its metabolites in hair? (3) What is the influence of hair's color? (4) Is there any racial bias in hair testing? (5) What is the influence of cosmetic treatments? Until now, the limiting factor for hair analysis in doping control is the lack of scientific data. The present article revises published material with special attention to the analytical and pharmacological parameters that may hinder the use of hair analysis in doping control.

UNITERMS: Hair analysis. Forensic toxicology. Doping control. Anabolics.

\section{AGRADECIMENTOS}

Ao Conselho Nacional de Desenvolvimento Científico e Tecnológico (CNPq). À Fundação Universitária José Bonifácio (FUJB). À Confederação Brasileira de Futebol (CBF).

\section{REFERÊNCIAS BIBLIOGRÁFICAS}

ABRAMSON, F. P., OSBON, B. L., TEFFERA, Y. Isotopic differences in human growth hormone preparations. Anal. Chem., v. 68, p. 1971-1972, 1996.

AQUINO NETO, F. R. O papel do atleta na sociedade e o controle de dopagem no esporte. Rev. Bras. Med. Esporte, vol. 7, p. 1-11, 2001. 
AUDRAN, M., KRAFFT, M-P., CEAURRIZ, J., MATHURIN, J-C., SICART, M-T., MARION, B., FABRE, F., BRESSOLLE, F. Assay method for perfluorooctyl bromide (perflubrom) in rat blood by gas chromatography-mass spectrometry. J. Chromatog. $B$, v. 734, p. 267-276, 1999.

AUGENSTEIN, W. M. Applied gas chromatography coupled to isotope ratio mass spectrometry. $J$. Chromatog. A, v. 842, p. 351-371, 1999.

AYOTTE, C., GOUDEAULT, D., CHARLEBOIS, A. Testing for natural and synthetic anabolic agents in human urine. J. Chromatog. B, v. 687, p. 3-26, 1996.

BAUMGARTNER, W. A., HILL, V.A. Hair analysis of drug abuse. Forensic Sci. Int., v. 63, p. 121-135, 1993.

BIRKILAND, K. I., HEMMERSBACH, P. The future of doping control in athletes: Issues related to blood sampling. Sports Med., v. 28, p. 25-33, 1999.

BÔER, T., BIJMA, R., ENSING, K. Modeling of conditions for the enantiomeric separation of $\beta 2$-adrenergic sympathicomimetics by capillary electrophoresis using cyclodextrins as chiral selectors in a polyethylene glycol gel. J. Pharmac. Biomed. Anal., v.19, p. 529-537, 1999.

BREO, D. L. OFMDs and muscles - lessons from two "retired steroids doctors". JAMA. J. Am. Med. Ass., v. 263, p. 1697, 1990.

BROWNE, A., LACHANCE, V., PIPE, A. The ethics of blood testing as an element of doping control in sport. Med. Sci. Sports Exerc., v.31, p. 497-501, 1999.

CALLIES, F., WIEBKILI, A., SIEKMAN, L., HUBLER, D., BIDLINGER, F., ALLOLIO, B. Influence of oral dehydroepiandrosterone (DHEA) on urinary steroid metabolites in males and females. Steroids, v. 65, p. 98$102,2000$.

CARRASQUILLA, H. M. Gas chromatography analysis of b2-agonists in bovine retina. Anal. Chem. Acta, v.408, p.285-290, 2000.

CARRASQUILLA, M. H. Gas chromatography-mass spectrometry analysis of anabolic compounds in bovine hair: evaluation of hair extraction procedures. Anal. Chem. Acta, v. 434, p. 59-66, 2001.
CHIAROTTI, M., STRANO-ROSSI, S., OFFIDANI, C., FIORI A. Evaluation of cocaine use during pregnancy through toxicological analysis of hair. J. Anal. Toxicol., v. 20 , p. $1-4,1996$.

CIRIMELE, V., KINTZ, P., JEANNEAEAU, T., LUDES, B. Testing for anabolic steroids in human hair obtained from two bodybuilders. Toxicoram., v. 11, p. 30-34, 1999a.

CIRIMELE, V., KINTZ, P., JEANNEAEAU, T., LUDES, B. Testing for anabolic steroids in human hair by gaschromatography-negative ion chemical ionization mass spectrometry. J. Chromatog. B, v. 740, p. 265-271, 2000.

CIRIMELE, V. Drug concentrations in human hair after bleaching. J. Anal. Toxicol., v. 19, p. 331, 1995.

CIRIMELE, V., KINTZ, P., JAMEY, C., LUDES, B. Are cannabinoids detected in hair after washing with Cannabiol Shampoo? J. Anal. Toxicol., v. 23, p. 349-351, 1999b.

CIRIMELE, V., SACHS, H., KINTZ, P., MANGIN, P. Testing human hair for cannabis. III. Rapid screening procedure for the simultaneous identification of D9tetrahydrocannabinol, cannabinol and cannabidiol. $J$. Anal. Toxicol., v. 20, p. 13-16, 1996.

CONE, E. J. Legal, workplace, and treatment drug testing with alternate biological matrices on global scale. Forensic Sci. Int., v.121, p. 7-15, 2001.

CONE, E. J. Mechanism of drug incorporation into hair. Ther. Drug Monit., v. 18, p. 438-443, 1996.

CONE, E. J. Saliva testing for drugs abuse. Ann. New York Acad. Sci., v. 694, p. 91-127, 1993.

CONE, E. J., HILLSGROVE, M. J., JENKINS, A. J., KEENAN, R. M., DARWIN, W. D. Sweat testing for heroin, cocaine and metabolites. J. Anal. Toxicol., v. 18 298-305, 1994.

CONE, E. J., DARWIN, W. D., WANG, W. L. The occurrence of cocaine, heroin metabolites in hair of drugs abusers. Forensic Sci. Int., v. 63, p. 55-68, 1993.

DE MERODE, A. Criteria to be applied to the quantitation and confirmation of anabolic steroids. COI guidelines. Lousanne, $1998.8 \mathrm{p}$. 
DE MERODE, A. Introduction and background. in doping. An IOC White Paper, IOC. Lousanne, 1999. 8.p

DeCOCK, K. J. S., DELBEKE, F. T., VAN EENOO, P., DESMET, N., ROELS, K., DE BACKER, P. Detection and determination of anabolic steroids in nutritional supplements. J. Pharmac.. Biomed. Anal., v. 25, p. 843852, 2001.

DELBEKE, F.T. Doping in ciclysm: results of unannounced controls in Flanders. Int. J. Sports Med., v. 17, p. 139, 1991.

DENG, X. S., KUROSU, A., POUNDER, D. J. Detection of anabolic steroids in head hair. J. Forensic Sci., v. 44, p. 343-346, 1999.

DEVEAUX, M., KINTZ, P., GOULLE, J. P., BESSARD, J., PEPIN, G., GOSSET, D. The hair analysis proficiencytesting program of the French society of analytical toxicology. Forensic Sci. Int., v. 107, p. 389-394, 2000.

DONGMI, C., MYUNGSOO, K., PARK, J. Erytropoietin: physico- and biochemical analysis. J. Chromatog. B, v. 687, p. 189-199, 1996.

DONIKE, M., RAUTH, S., WOLANSKY, A. References ranges of urinary endogenous steroids determined by gas chromatography-mass spectrometry. In: COLOGNE WORKSHOP ON DOPE ANALYSIS, 10., 1992, Cologne. Proceedings. Cologne: Sport and Buch Straub, 1992. p. 69-75.

DRAISCI, R., PALLESCH, L., MARCHIAFAVA, C., FERRETI, E., QUADRI, F.D. Confirmatory analysis of residues of stanozolol and its major metabolite in bovine urine by liquid chromatography-tandem mass spectrometry. J. Chromatog. A v. 926, p. 69-77, 2001.

FUCCI, N., DE GIOVANNI, N., CHIARROTI, M., SCARLATA, S. SPME-GC analysis of THC in saliva samples collected with "EPITOPE" device. Forensic Sci. Int., v.119, p. 318-321, 2001.

GAILLARD, Y., BALLAND, A., DOUCET, F., PEPIN, G. Detection of illegal use of clembuterol in calves using hair analysis. Applications in meat quality control. $J$. Chromatog. B, v. 730, p. 85-95, 1997.
GAILLARD, Y., VAYSSETTE, F., BAILLAND, A., PEPIM, G. Gas chromatographic-tandem-mass spectrometric determination of anabolic steroids and their esters in hair application in doping control and meat quality. J. Chromatog. B, v. 735, p. 189-205, 1999a.

GAILLARD, Y., VAYSSETTE, F., PEPIM, G. Compared interest between hair analysis and urinalysis in doping control: results for amphetamines, corticosteroids and anabolic steroids in racing cyclists. In: EUROPEAN MEETING ON HAIR ANALYSIS, 2., 1999. Proceedings. Martiny: Bundesinstituit für sportwissenshaft,1999b. p. 14-16.

GAILLARD, Y., VAYSSETTE, F., PEPIM, G. Compared interest between hair analysis and urinalysis in doping control: results for amphetamines, corticosteroids and anabolic steroids in racing cyclists. Forensic Sci. Int., v.107, p. 361-379, 2000.

GEYER, H., MARECK-ENGELKE, U., SCHÄNZER, W. The analysis of nutritional supplements for anabolic androgenic steroids. In: RECENT ADVANCES IN DOPING CONTROL, 8., COLOGNE WORKSHOP ON DOPE ANALYSIS, 18., Cologne, 2000. Proceedings. Cologne: Sport and Buch Straub, 2001. p. 23-32.

GEYER, H., SCHÄNZER, W., MARECK-ENGELKE. U., M. DONIKE. Sample purification of urine samples for improved detection of anabolic and endogenous steroids. In: RECENT ADVANCES IN DOPING CONTROL, 6 , COLOGNE WORKSHOP ON DOPE ANALYSIS, 15. Cologne, 1997. Proceedings. Cologne: Sport and Buch Straub, 1998. p. 97-104.

GLEIXNER, A., SAUERWEIN, H., MEYER, H. H. D. Detection of the anabolic b2-adrenoceptor agonist clenbuterol in human scalp hair by HPLC/EIA. Clin. Chem., v. 42, p. 1869-1871, 1996.

GRAHAM, K., KOREN, G., KLEIN, J., SCHENEIDERMAN, J., GRENWALD, M. Determination of gestational cocaine exposure by hair analysis. JAMA. J. Am. Med. Ass., v. 262, p. 3328-3330, 1989.

HEALY, R. W., RUSSEL, J. D. Growth hormone and sport : abuse, potential benefits, and difficulties in detection. $\mathrm{Br}$. J. Sports Med., v. 31, p. 267-268, 1997. 
HEMMERSBACH, P., DE LA TORRE, R. Stimulants, narcotics and beta-blockers: 25 years of developments in analytical analytical techniques for doping control. $J$. Chromatog. B, v. 687, p. 221-238, 1996.

HENDERSON, G. L. Mechanisms of drug incorporation into hair. Forensic Sci. Int., v.63, p. 19-29, 1993.

HENDERSON, G. D., HARKEY, M. R., ZHOU, C., JONES, R. T., JACOB III, P. Incorporation of isotopically labeled cocaine and metabolites into hair: race as a factor. J. Anal. Toxicol., v. 22, p. 156-165, 1998.

HENDERSON, G. L., HARKEY, M. R., ZHOU, C.; JONE, R. T., JACOB III, P. Incorporation of isotopically labeled cocaine and metabolites into human hair: 1. Doseresponse relationships. J. Anal. Toxicol., v. 20, p.1-12, 1996.

HENZE, M. K., OPFERMANN, G., LANGGUTH, H. S., SCHÄNZER, W. Screening of b2-agonists and confirmation of fenoterol, orciprenalina, reproterol and terbutalina with gas chromatography-mass spectrometry as tetrahydroisoquinoline derivatives. J. Chromatog. B, v.751, p. 93-105, 2001.

HOLD, K. M., WILKINS, D. G., CROUCH, D. J., ROLLINS, D. E., MÃES, R. A. Detection of stanozolol in hair by negative ion chemical ionization mass spectrometry. J. Anal. Toxicol., v. 20 p. 345-349, 1996.

HORNING, M. G., BROWN, L., NOWLIN, J., LERTRATANANGKOON, P. K., KELLAWAY, P., ZION, T. E. Use of saliva in therapeutic drug monitoring. Clin. Chem., v.23, p. 157-164, 1977.

HORNING, S., GEYER, H., FLENKER, U., SHANZER, W. Detection of exogenous Steroids by ${ }^{13} \mathrm{C} /{ }^{12} \mathrm{C}$ analysis. In: RECENT ADVANCES IN DOPING CONTROL, 6 ., COLOGNE WORKSHOP ON DOPE ANALYSIS, 15. 1997, Cologne. Proceedings. Cologne: Sport and Buch Straub, 1998b. p.135-148.

HORNING, S., SCHÄNZER, W., SAMPLE, B. HRMS analyses performed at the 1996 summer Olympic Games. In: RECENT ADVANCES IN DOPING CONTROL, 6., COLOGNE WORKSHOP ON DOPE ANALYSIS, 15., Cologne, 1997. Proceedings. Cologne: Sport and Buch Straub, 1998. p. 329-337.
HOWELLS, L., GODFREY, M., SAUER, M. J. Melanin as an adsorbent for drug residues. Analyst, v. 119, p. 2691$2693,1994$.

IOC, Olympic Movement Anti-doping Code, I.O.C., Lousanne, Switzerland, 1999.

JURADO, C., KINTZ, P., MENENDEZ, M., REPPETO, M. Influence of cosmetic treatment of hair on drug testing. Int. J. Leg. Méd., v. 110, p. 159-163, 1997.

KIDWELL, D.A. Is the hair testing culturally biased and why is this concern in the USP? In: EUROPEAN MEETING ON HAIR ANALYSIS, 2.,1999. Proceedings. Martiny: Bundesinstituit für sportwissenshaft, 1999. p. 14-16.

KINTZ, P., CIRIMELE, V., DUMESTRE, T. V., LUDES, B. Doping control of nandrolone using hair analysis. $J$. Pharmac. Biom. Anal., v. 24, p. 1125-1130, 2001.

KINTZ, P. Clinical applications of hair analysis. In: Drug testing in hair. Boca Raton: CRC Press, 1996a. p. 267-277.

KINTZ, P. Hair analysis of nordazepam and oxazepam by gas chromatography-negative-ion chemical ionization mass spectrometry. J. Chromatog. B, v. 667, 241-244, 1996 b.

KINTZ, P. Hair testing and doping control. Toxicol. Lett., v. 102-103, p. 109-113, 1998.

KINTZ, P., CIRIMELE, V., LUDES, B. Physiological concentrations of DHEA in human hair. J. Anal. Toxicol., v. 23, p. $424-428,1999$ a.

KINTZ, P., CIRIMELE, V., SACHS, H., JEANNEAU, T., LUDES, B. Testing steroids in hair of two bodybuilders. Forensic Sci. Int., v. 101, p. 209-216, 1999 b.

KINTZ, P., CIRIMELE, V., JEANNEAU, T., LUDES, B. Identification of testosterone and testosterone esters in human hair. J. Anal. Toxicol., v. 23, p. 352-356, 1999c.

KINTZ, P., MANGIN, P. Evidence of gestational heroin or nicotine exposure by analysis of fetal hair. Forensic Sci. Int., v.70, p. 99-104, 1993.

KINTZ, P., MANGIN, P. Simultaneous determination of opiates, cocaine and major metabolites of cocaine in human hair by GC/MS. Forensic Sci. Int., v.73, p. 93100, 1995. 
KINTZ, P., SAMYN N. Determination of "Ecstasy" components in alternative biological specimens. $J$. Chromatog. B, v. 733, p. 137-143, 1999.

KINTZ, P., TRACQUI, A., MANGIN, P. Detection of drugs in human hair for clinical and forensic applications. Int. J. Leg. Med., v.105, p. 1-4, 1992.

KIRCHER, V., PARLAR, H. Determination of delta-9tetrahydrocannabinol from human saliva by tandem immunoafinity chromatography high performance liquid chromatography. J. Chromatog. Appl., v. 677, p. 245255, 1996.

KRAFFT, M. P. Fluorocarbons and fluorinated amphiphiles in drug delivery and biomedical research. Adv. Drug Deliv. Rev., v. 47, p. 209-228, 2001.

KRONSTRAND, R., FORSBERG, S., KAGEDAL, B., AHLNER, J., LARSON, G. Relationship between melanin and codeine concentrations in hair after oral administration In: PROCEEDINGS OF THE ANNUAL MEETINGS OF THE AMERICAN ACADEMY OF FORENSIC SCIENCES, Orlando, 1999. Proceedings. Orlando: Academic Press,1999. Abstract K12.

LAC, G., LAC. N., ROBERT, A. Steroid assays in saliva: a method to detect plasmatic contamination. Arch. Int. Physiol. Biochem. Biophy., v. 101, p.257-262. 1993.

LAURE, P. General practitioners and doping in sports: knowledge and attitudes. Sante Publique, v. 9, p.145-156, 1997.

MACHNICK, M., GEYER, H., HORNING, S., BREDBACH, A., DELAHAUT, P., SCHÄNZER, W. Long-term detection of clembuterol in human scalp hair by gas chromatography-high resolution mass spectrometry. J. Chromatog. B, v. 723, p. 147-155, 1999.

MADEA, B., GREENER, W., MUSSOF, F, DETTMEYER, R. Medico-legal aspects of doping. J. Clin. Forensic Med., v. 5, p. 1-7, 1998.

MARCH, A., EVANS, M. B. RIA of drugs of abuse in hair. Part I: methadone in human hair, method adoption and the evaluation of decotamination procedures. J. Pharm. Biomed. Anal., v. 12, p. 1123-1130, 1994.
MARQUES, M. A. S., BIZARRI, C. H. B., CARDOSO, J. N., AQUINO NETO, F. R. Effect of finasteride on the urinary steroid profile: a case study. In: COLOGNE WORKSHOP ON DOPE ANALYSIS, 7., Cologne 1999. Proceedings. Cologne: Sport and Buch Straub, 1999. p. 317-322.

MOLLER, M. R. Hair analysis as evidence in forensic cases. Drug Monit., v.18, p. 444-449, 1996.

MOLLER, M.R., FEY, P. Identification and quantitation of cocaine and its metabolites, benzoylecgonine and ergonine methyl esters, in hair of Bolivian coca chewers by gas chromatography/ Mass spectrometry. J. Anal. Toxicol., v. 19, p. 291-296, 1992.

NAKAHARA, Y. Detection and diagnostic interpretation of amphetamines in hair. For. Sci. Int., v.70, p.135-153, 1995.

NAKAHARA, Y., TAKAHASCHI, K., KIKURA, R. Hair analysis for drugs of abuse. X. Effects of physiochemical properties of drugs on the incorporation rates into the hair. Biol. Pharm. Bull., v. 18, p. 1233-1227, 1995.

OFFIDANI, C. Drug distribution in the head, axillary and pubic of chronics addicts. Forensic Sci. Int., v.63, p.105108, 1993.

PEPIN, G., GAILLARD, Y. Concordance between selfreported drug use and findings in hair about cocaine and heroin. Forensic Sci. Int., v. 84, p. 37-41, 1997.

PEREIRA, H. M. G., MARQUES, M. A. S., CARDODO, J. N., AQUINO NETO, F. R. Derivatization study on endogenous and synthetic corticosteróides by gas chromatography mass spectrometry. In: RECENT ADVANCES IN DOPING ANALYSIS, 9., MANFRED DONIKE WORKSHOP, COLOGNE WORKSHOP ON DOPE ANALYSIS, 19., Cologne, 2001. Proceedings. Cologne: Sport and Buch Straub, 2002. p. 203-207.

PERKONING, A., LIEB, R., WITTCHER, H. U. Prevalence of abuse and dependence of illicit drugs among adolescents and young adults in a community sample. Eur. Addict. Res., v. 4, p. 5-66, 1998.

POTSCH, L., SKOPP, G. Stability of opiates in hair fibers exposure to cosmetic treatment. Forensic Sci. Int., v. 81, p. 95-102, 1996. 
PRAGST, F., ROTHE, M., SPIEGEL, K., SPORKEST, F. Illegal and therapeutic drug concentrations in hair segments - a timetable of drug exposure. Forensic Sci. Rev., v. 10, p. 81-111, 1998.

PRESTON, K. L., SILVERMAN, K., CSHUSTER C. R., CONE, E. J. Assesment of cocaine use with quantitative urianalysis and estimation of new uses. Addic. v.92, p. 717-727, 1997.

QUINTELLA, O., BERNEJO, A. M., TABERNERO, M. J., STRANO-ROSSI, S., CHIAROTTI, M., LUCAS, A. C. $\mathrm{S}$. Evaluation of cocaine, amphetamines and cannabis use in university students through hair analysis: preliminary results. Forensic Sci. Int., v. 107, p. 273-279, 2000 .

RAMOS, F., SANTOS, C., SILVA, A., SILVEIRA, M. I. N. b2-adrenergics agonists residues: simultaneous methyland buthylboronic derivatization for confirmatory analysis by gas chromatography-mass spectrometry. $J$. Chromatog. B, v. 716, p. 366-370, 1998.

RAMOS, F. J. $\beta_{2}$-Agonists extraction procedures for chromatografic analysis. J. Chromatog. A, v. 880, p. 6983,2000 .

RICKERT, V. I., PAVLACK, M. C., SHEPPARD, V., JAY, M. S. Human growth hormone: a new substance of abuse among adolescent?. Clin. Pediat., v. 31, p.723-726, 1992.

RIESS, J. G. Fluorocarbons-based in vivo oxygen transport and delivery systems. Vox Sang., v. 61, p. 225-239, 1999.

RIVIER, L. Is there a place for hair analysis in doping control? Forensic Sci. Int., v. 107, p. 309-323, 2000.

ROLLINS, D. E., WILKINS, D. G., KRUEGER, G. G. Codeine disposition in human after signal and multiple doses. Eur. J. Clin. Pharmacol., v. 50, p. 391-397, 1996.

SAUGY, M. Hormone de croissance et sport. Rev. Franc. Des. Laborat., v. 331, p.45-49, 2001.

SAUGY, M., CARDIS, C., SCHWEIZER, C., VEUTHEY, J. L., RIVIER, L. Detection of human growth hormone doping in urine: out of competition are necessary. $J$. Chromatog. B, v. 687, p. 201-211, 1996.
SAWAYA, W. N., LONE, K., HUSSAIN, A., DASHTI, B., SAEED, T. Screening for $\beta 2$-agonists in sheep urine and eyes by an enzymatic-linked immunosorbent assay in the state of Kuwait. Food Control, v.11, p.1-5, 2000.

SCHÄNZER, W. Dopinganalitik. Institut für Biochemie. Disponível em: $<$ http://www.dshs.koeln.de/biochemie/ rubriken/07 info/analitik.pdf $>$. Acesso em: 17 out. 2001.

SCHÄNZER, W., BREDBACK, A., GEYER, H., KUK, C. V., NOLTEERSNSTING, E. Metabolism of nortestosterone, norandrosterone and norandrostenediol: Identification of $3 \alpha$-hydroxyestr-4-en-17-one glucuronide and $3 \alpha, 16 \alpha$-dihydroxy-5 $\alpha$-estran-17-one glucuronide and sulphate. In: RECENT ADVANCES IN DOPING ANALYSIS, 8., MANFRED DONIKE WORKSHOP, COLOGNE WORKSHOP ON DOPE ANALYSIS, 18., Cologne, 2000. Proceedings. Cologne: Sport and Buch Straub, 2001. p. 155-174.

SCHÄNZER, W., DELAHAUT, P., GEYER, H., MACHINIK, M., HORNING, S. Long term detection and identification of metandienone and stanozolol abuse in athletes by gas chromatography high resolution mass spectrometry. J. Chromatog. B, v. 687, p.93-70, 1996.

SCHÄNZER, W., DONIKE, M. Metabolism of anabolic steroids in man: synthesis and use of reference substances for identification of anabolic steroid metabolites. Anal. Chim. Acta., v. 275, p. 23-48, 1993.

SCHRAMAN, W., SMITH, R.H., CRAIG, P.A. Drugs abuse in saliva: a review. J. Anal. Toxicol., v. 16, p. 1-9, 1992.

SEGURA, J., PICHINI, S., PENG, S.H., DE LA TORRE, X. Hair analysis and detection of single dose administration of androgenic steroids esters. In: EUROPEAN MEETING ON HAIR ANALYSIS, 2., Martiny, 1999. Proceedings. Martiny: Bundesinstituit für sportwissenshaft, 1999.p. 16

SHACKELTON, C. H. L., ROITMAN, E., PHILLIPS, A., CHANG, T. Androstenediol and 5-androstenediol profiling for detecting exogenously administered dihydrotestosterone, epitestosterone and dehydroepitestosterone. Potential use in gas chromatography isotope ratio mass spectrometry. Steroids, v. 62, p. 665-673, 1997.

SHERER, G., WACHTER, U., WUDY, S. A. Determination of testosterone in human hair by gas chromatography selected ion monitoring mass spectrometry. Analyst, v. 123, p. 2661-2663, 1998. 
SPHON, R. Use of mass spectrometry for confirmation of minimal drug diseases. J. Assoc. Anal. Chem., v. 5, p. 1247-1251, 1961.

SPRINGFIELD, A. C., CARTMELL, L. W., AUFDERHEIDE, A. C., BUJKSTRA, J., HO, J. Cocaine metabolites in the hair of ancient coca leaf chewers. Forensic Sci. Int., v. 63, p. 269-275, 1993.

STAUB, C. Hair analysis: its importance for the diagnosis of poisoning associated with opiate addiction. Forensic Sci. Int., v. 63, p. 69-75, 1993.

STEPHANY, R. W., VAN GINKEL, L. A. Quality criteria for residue analysis and reference materials. Relationship between legal procedures and materials. Fresenius $J$. Anal. Chem., v. 338, p. 370-377, 1990.

SWINKLES, L. M. J. W., VAN HOOF, H. J. C., ROSS, H. A., SMALS, A. G. H., BENRAAD, T. J. Low ratio of androstenedione to testosterone in plasma and saliva of hirsute women. Clin. Chem., v.38/39, p. 1819-1823, 1992.

The OMAC 2001. Olympic Movement Anti-doping Code. Appendix A. 4.

THEVIS, M., OPFERMANN, G., SCHÄNZER, W. Detection of the plasma expander hydroxyethyl starch in human urine. J. Chromatog. B., v. 744, p. 345-350, 2000.

THIEME, D., GROSSE, J., SACHS, H., MUELLER, R. K. Detection of several anabolic steroids of abuse in human hair. In: RECENT ADVANCES IN DOPING ANALYSIS, 7., COLOGNE WORKSHOP ON DOPE ANALYSIS, 16., Cologne 1998. Proceedings. Cologne: Sport und Buch, Koln, 1999. p. 9-29.
TRACQUI, A. Hair analysis a worthless tool for therapeutic compliance monitoring. Forensic Sci. Int., v.70, p. 183189, 1995.

TRACQUI, A., KINTZ, P., MAGUN, P. HPLC/MS determination of buprenorphine and norbuprenorphine in biological fluids and hair samples. J. Forensic. Sci., v. 42, p. 111-114, 1997.

UEMATSU,T., SATO, R. Human scalp hair as evidence of individual dosage history of haloperidol: long-term follow-up study. Ther. Drug. Monitor., v. 12, p. 582-583, 1990.

VERSTRAET, A. G., PIERCE, A. Workplace drug testing in Europe. Forensic. Sci. Int., v. 121, p. 2-6, 2001.

VICENT, F., BESSARD, J., VACHERON, J., MALLARD, M., BESSARD, G. Determination of buprenorphine and norbuprenorphine in urine and hair by GC-MS. J. Anal. Toxicol., v. 23, p. 270-279, 1999.

WENNING, R. Potential problems with the interpretation of hair analysis results. Forensic Sci. Int., v. 107, 5-12, 2000.

WHEELER, M. J., ZHONG, Y. B., KIEMAN, A. T., COUTTS, S. B. The measurements of testosterone in hair. J. Endocrinol., v. 159, p. R5-R8, 1998.

WU, A. H. B., HILL, D. H., CROUCH, D., HODNETT, C. N., MCCURDY, H. H. Minimal standards for the performance and interpretation of toxicology tests in legal proceedings. J. Forens. Sci., v.44, p. 516-522, 1999.

Recebido para publicação em 05 de novembro de 2001. 